\title{
Dilaton Black Holes in de Sitter or Anti-de Sitter Universe
}

\author{
Chang Jun Gao ${ }^{1 *}$ Shuang Nan Zhang ${ }^{1,2,3,4 \dagger}$ \\ ${ }^{1}$ Department of Physics and Center for Astrophysics, Tsinghua University, Beijing 100084, \\ China(mailaddress) \\ ${ }^{2}$ Physics Department, University of Alabama in Huntsville, AL 35899, USA \\ ${ }^{3}$ Space Science Laboratory, NASA Marshall Space Flight Center, SD50, Huntsville, AL 35812, \\ $U S A$ \\ ${ }^{4}$ Laboratory for Particle Astrophysics, Institute of High Energy Physics, Chinese Academy of \\ Sciences, Beijing 100039, China
}

(July 23, 2018)

\begin{abstract}
Poletti and Wiltshire have shown that, with the exception of a pure cosmological constant, the solution of a dilaton black hole in the background of de Sitter or anti-de Sitter universe, does not exist in the presence of one Liouville-type dilaton potential. Here with the combination of three Liouvilletype dilaton potentials, we obtain the dilaton black hole solutions in the background of de Sitter or anti-de Sitter universe.
\end{abstract}

PACS number(s): 04.20.Ha, 04.50.+h, 04.70.Bw

*E-mail: gaocj@mail.tsinghua.edu.cn

${ }^{\dagger}$ E-mail: zhangsn@mail.tsinghua.edu.cn 


\section{INTRODUCTION}

Dilaton is a kind of scalar field occurring in the low energy limit of the string theory where the Einstein action is supplemented by fields such as axion, gauge fields and dilaton coupling in a nontrivial way to the other fields. Exact solutions for charged dilaton black holes in which the dilaton is coupled to the Maxwell field have been constructed by many authors. It is found that the presence of dilaton has important consequences on the causal structure and the thermodynamic properties of the black hole [1-9]. Thus much interest has been focused on the study of the dilaton black holes.

On the other hand, there has also been some renewed interest in the study of black hole theories with the cosmological constant. Theories with negative cosmological constant can be embedded in a supersymmetric setting in which gauged supergravity theories are obtained in various dimensions. Gauged supergravities admit anti-de Sitter spacetime as a vacuum state, and thus black hole solutions of these theories are of physical relevance to the proposed AdS/CFT correspondence [10-15]. In particular, the study of AdS black holes can give new insights into the nonperturbative structure of some conformal field theories. Black holes in the background with positive cosmological constant, i.e., in de Sitter spacetime, have also attracted some interest recently, due to the phenomenon of black hole anti-evaporation [16]. Besides, these black holes could be relevant to the proposed duality between the large N limit of Euclidean four-dimensional U(N) super-Yang-Mills theory and the so-called type IIB. string theory in de Sitter spacetime [17]. So the object of the present paper is to find the dilaton black hole solutions in the de Sitter or anti-de Sitter spacetime.

In fact, Poletti and Wiltshire [18] have shown that with the exception of a pure cosmological constant, no dilaton-de Sitter or anti-de Sitter black hole solution exists with the presence of only one Liouville-type dilaton potential. Okai, who made investigations using power series [19], has been unable to prove unequivocally that dilaton-de Sitter or anti-de Sitter black hole solutions do exist. In this paper, with the combination of three Liouvilletype dilaton potentials, the solutions of dilaton black holes in the background of de Sitter 
or anti-de Sitter spacetime are achieved.

The paper is organized as follows. In Sec.2, we will derive the solution in the cosmic coordinate system with a new method developed recently by us. In Sec.3, we find the coordinates transformation which recast the solution in the schwarzschild coordinates system. In Sec.4, we deduce the dilaton potential with respect to the cosmological constant. In Sec.5 and Sec.6, we generalize the solution to arbitrary coupling constant and arbitrary number black holes cases. In Sec.7, the horizon property of the black hole is discussed. We conclude with some final remarks.

\section{DILATON-DE SITTER METRIC IN COSMIC COORDINATE SYSTEM}

The metric of a dilaton black hole in the Schwarzschild coordinate system is given by

$$
d s^{2}=-\left(1-\frac{2 M}{x}\right) d t^{2}+\left(1-\frac{2 M}{x}\right)^{-1} d x^{2}+x(x-2 D) d \Omega_{2}^{2}
$$

where $M$ and $D$ are the mass and dilaton charge of the black hole, respectively. $D$ is related to the mass $M$ and electric charge $Q$ as follows

$$
D=\frac{Q^{2} e^{2 \phi_{0}}}{2 M}
$$

$\phi_{0}$ is the asymptotic constant value of the dilaton.

In order to obtain the dilaton-de Sitter metric, we should rewrite the metric Eq.(1) in the cosmic coordinate system. So make variable transformation $x \rightarrow r$

$$
x=\frac{(r+M+D)^{2}-4 M D}{2 r},
$$

then we can rewrite Eq.(1) as follows

$$
\begin{aligned}
d s^{2}= & -\frac{\left(1-\frac{M}{r}+\frac{D}{r}\right)^{2}}{\left(1+\frac{M}{r}+\frac{D}{r}\right)^{2}-\frac{4 M D}{r^{2}}} d t^{2} \\
& +\frac{1}{4}\left(1+\frac{M}{r}-\frac{D}{r}\right)^{2}\left[\left(1+\frac{M}{r}+\frac{D}{r}\right)^{2}-\frac{4 M D}{r^{2}}\right]\left(d r^{2}+r^{2} d \Omega_{2}^{2}\right) .
\end{aligned}
$$


Re-scale the variables $t$ and $s$, we have

$$
\begin{aligned}
d s^{2}= & -\frac{\left(1-\frac{M}{r}+\frac{D}{r}\right)^{2}}{\left(1+\frac{M}{r}+\frac{D}{r}\right)^{2}-\frac{4 M D}{r^{2}}} d t^{2} \\
& +\left(1+\frac{M}{r}-\frac{D}{r}\right)^{2}\left[\left(1+\frac{M}{r}+\frac{D}{r}\right)^{2}-\frac{4 M D}{r^{2}}\right]\left(d r^{2}+r^{2} d \Omega_{2}^{2}\right)
\end{aligned}
$$

Following the method we developed recently [20], we make the following replacements

$$
1 \longrightarrow a^{\frac{1}{2}}, \quad \frac{M}{r} \longrightarrow \frac{M}{r a^{\frac{1}{2}}}, \quad \frac{D}{r} \longrightarrow \frac{D}{r a^{\frac{1}{2}}},
$$

where $a \equiv e^{H t}, H$ is a constant which has the meaning of Hubble constant. Then the dilaton-de Sitter metric is achieved

$$
\begin{aligned}
d s^{2}= & -\frac{\left(1-\frac{M}{a r}+\frac{D}{a r}\right)^{2}}{\left(1+\frac{M}{a r}+\frac{D}{a r}\right)^{2}-\frac{4 M D}{a^{2} r^{2}}} d t^{2} \\
& +a^{2}\left(1+\frac{M}{a r}-\frac{D}{a r}\right)^{2}\left[\left(1+\frac{M}{a r}+\frac{D}{a r}\right)^{2}-\frac{4 M D}{a^{2} r^{2}}\right]\left(d r^{2}+r^{2} d \Omega_{2}^{2}\right) .
\end{aligned}
$$

Eq.(7) is just the charged dilaton black hole solution in the background of de Sitter universe in the cosmic coordinate system. When $M=D=0$, it recovers the well-known de Sitter metric. On the other hand, when $H=0$, it recovers the charged dilaton metric Eq.(5). In the next section, we rewrite it in the Schwarzschild coordinate system by coordinates transformation.

\section{DILATON-DE SITTER OR ANTI-DE SITTER METRICS IN SCHWARZSCHILD COORDINATE SYSTEM}

In order to present the dilaton-de Sitter metric in the Schwarzschild coordinate system, we make variable transformation $r \rightarrow y$ as follows

$$
r=a^{-1}[y-M-D+\sqrt{(y-2 M)(y-2 D)}] .
$$

Eq.(7) becomes 


$$
\begin{aligned}
d s^{2}= & -\left[1-\frac{2 M}{y}-4 y(y-2 D) H^{2}\right] d t^{2}+\frac{4}{1-\frac{2 M}{y}} d y^{2} \\
& -\frac{8 H \sqrt{y(y-2 D)}}{\sqrt{1-\frac{2 M}{y}}} d t d y+4 y(y-2 D) d \Omega_{2}^{2} .
\end{aligned}
$$

Re-scale $t$ and $s$, we have

$$
\begin{aligned}
d s^{2}= & -\left[1-\frac{2 M}{y}-4 y(y-2 D) H^{2}\right] d t^{2}+\frac{1}{1-\frac{2 M}{y}} d y^{2} \\
& -\frac{4 H \sqrt{y(y-2 D)}}{\sqrt{1-\frac{2 M}{y}}} d t d y+y(y-2 D) d \Omega_{2}^{2} .
\end{aligned}
$$

Eq.(10) has a $d t d y$ term. In order to eliminate this term, we introduce a new time variable $u$, namely, $t \rightarrow u$

$$
t=u-\int \frac{2 H \sqrt{y(y-2 D)}}{\sqrt{1-\frac{2 M}{y}}\left[1-\frac{2 M}{y}-4 y(y-2 D) H^{2}\right]} d y .
$$

Then Eq.(10) is reduced to

$$
\begin{aligned}
d s^{2}= & -\left[1-\frac{2 M}{y}-4 y(y-2 D) H^{2}\right] d u^{2}+\left[1-\frac{2 M}{y}-4 y(y-2 D) H^{2}\right]^{-1} d y^{2} \\
& +y(y-2 D) d \Omega_{2}^{2} .
\end{aligned}
$$

Let $H^{2}$ absorb the constant 4 and rewrite the variables $(t, r)$ instead of $(u, y)$, we obtain the dilaton-de Sitter metric in the Schwarzschild coordinate system

$$
\begin{aligned}
d s^{2}= & -\left[1-\frac{2 M}{r}-r(r-2 D) H^{2}\right] d t^{2}+\left[1-\frac{2 M}{r}-r(r-2 D) H^{2}\right]^{-1} d r^{2} \\
& +r(r-2 D) d \Omega_{2}^{2} .
\end{aligned}
$$

When $D=0$, it restores to the well-known Schwarzschild-de Sitter metric. On the other hand, when $H=0$, it restores to the charged dilaton metric which is found by Garfinkle, Horowitz and Strominger [2]. To show Eq.(13) does represent a solution of Einstein-Maxwelldilaton theory, in the following section, we will derive the potential of the dilaton for the cosmological constant. In order that the negative cosmological constant case is included in our solution, we make the replacement of $H^{2}=\lambda / 3$ in Eq.(13). $\lambda$ is the cosmological constant which can be positive or negative. 


\section{POTENTIAL OF THE DILATON FOR COSMOLOGICAL CONSTANT}

We consider the four-dimensional theory in which gravity is coupled to dilaton and Maxwell field with an action

$$
S=\int d^{4} x \sqrt{-g}\left[R-2 \partial_{\mu} \phi \partial^{\mu} \phi-V(\phi)-e^{-2 \phi} F^{2}\right]
$$

where $R$ is the scalar curvature, $F^{2}=F_{\mu \nu} F^{\mu \nu}$ is the usual Maxwell contribution, and $V(\phi)$ is a potential for $\phi$.

Varying the action with respect to the metric, Maxwell, and dilaton fields, respectively, yields

$$
\begin{gathered}
R_{\mu \nu}=2 \partial_{\mu} \phi \partial_{\nu} \phi+\frac{1}{2} g_{\mu \nu} V+2 e^{-2 \phi}\left(F_{\mu \alpha} F_{\nu}^{\alpha}-\frac{1}{4} g_{\mu \nu} F^{2}\right), \\
\partial_{\mu}\left(\sqrt{-g} e^{-2 \phi} F^{\mu \nu}\right)=0, \\
\partial_{\mu} \partial^{\mu} \phi=\frac{1}{4} \frac{\partial V}{\partial \phi}-\frac{1}{2} e^{-2 \phi} F^{2} .
\end{gathered}
$$

The most general form of the metric for the static space-time can be written as

$$
d s^{2}=-U(r) d t^{2}+\frac{1}{U(r)} d r^{2}+f(r)^{2} d \Omega_{2}^{2}
$$

Then the Maxwell equation Eq.(16) can be integrated to give

$$
F_{01}=\frac{Q e^{2 \phi}}{f^{2}}
$$

where $Q$ is the electric charge. With the metric Eq.(18) and the Maxwell field Eq.(19), the equations of motion Eqs.(15-17) reduce to three independent equations

$$
\begin{gathered}
\frac{1}{f^{2}} \frac{d}{d r}\left(f^{2} U \frac{d \phi}{d r}\right)=\frac{1}{4} \frac{d V}{d \phi}+e^{2 \phi} \frac{Q^{2}}{f^{4}} \\
\frac{1}{f} \frac{d^{2} f}{d r^{2}}=-\left(\frac{d \phi}{d r}\right)^{2},
\end{gathered}
$$




$$
\frac{1}{f^{2}} \frac{d}{d r}\left(2 U f \frac{d f}{d r}\right)=\frac{2}{f^{2}}-V-2 e^{2 \phi} \frac{Q^{2}}{f^{4}} .
$$

Substituted

$$
f=\sqrt{r(r-2 D)}, \quad U=1-\frac{2 M}{r}-\frac{1}{3} \lambda r(r-2 D),
$$

into Eqs.(20-22), we obtain the dilaton field, dilaton charge and dilaton potential

$$
\begin{aligned}
& e^{2 \phi}=e^{2 \phi_{0}}\left(1-\frac{2 D}{r}\right), \\
& D=\frac{Q^{2} e^{2 \phi_{0}}}{2 M}, \\
& V(\phi)=\frac{4}{3} \lambda+\frac{1}{3} \lambda\left[e^{2\left(\phi-\phi_{0}\right)}+e^{-2\left(\phi-\phi_{0}\right)}\right] .
\end{aligned}
$$

Compare these solutions to the result of Garfinkle, Horowitz and Strominger, we find that the Maxwell field, the dilaton filed and the dilaton charge of the (anti) de Sitter versions

are exactly identical to that of the dilaton black hole. The potential of the dilaton is the combination of a constant and two Liouville-type potentials.

So far, we obtained the action of the dilaton-(anti) de Sitter black hole

$$
S=\int d^{4} x \sqrt{-g}\left\{R-2 \partial_{\mu} \phi \partial^{\mu} \phi-\frac{4}{3} \lambda-\frac{1}{3} \lambda\left[e^{2\left(\phi-\phi_{0}\right)}+e^{-2\left(\phi-\phi_{0}\right)}\right]-e^{-2 \phi} F^{2}\right\} .
$$

When $\phi=\phi_{0}=0$, it reduces to the action of Reissner-Nordström-de Sitter black hole.

\section{DILATON-(ANTI) DE SITTER METRIC WITH ARBITRARY $\alpha$}

Up to now, we have only dealt with the dilaton-(anti) de Sitter metric for the case that the coupling constant $\alpha=1$. In this section, we will present the dilaton-(anti) de Sitter metric with an arbitrary coupling constant. Following the method adopted above, we obtain the dilaton-(anti) de Sitter metric with an arbitrary coupling constant $\alpha$ in the cosmic coordinate system

$$
\begin{aligned}
d s^{2}= & -\left(1-\frac{r_{+}}{a r}+\frac{r_{-}}{a r}\right)^{2}\left(1+\frac{r_{+}}{a r}-\frac{r_{-}}{a r}\right)^{\frac{2\left(1-\alpha^{2}\right)}{1+\alpha^{2}}}\left[\left(1+\frac{r_{+}}{a r}+\frac{r_{-}}{a r}\right)^{2}-\frac{4 r_{+} r_{-}}{a^{2} r^{2}}\right]^{\frac{-2}{1+\alpha^{2}}} d t^{2} \\
& +a^{2}\left(1+\frac{r_{+}}{a r}-\frac{r_{-}}{a r}\right)^{\frac{4 \alpha^{2}}{1+\alpha^{2}}}\left[\left(1+\frac{r_{+}}{a r}+\frac{r_{-}}{a r}\right)^{2}-\frac{4 r_{+} r_{-}}{a^{2} r^{2}}\right]^{\frac{2}{1+\alpha^{2}}}\left(d r^{2}+r^{2} d \Omega_{2}^{2}\right)
\end{aligned}
$$


where $a=e^{H t}$ is the scale factor of the universe and the equivalent form in Schwarzschild coordinate system

$$
\begin{aligned}
d s^{2}= & -\left[\left(1-\frac{r_{+}}{r}\right)\left(1-\frac{r_{-}}{r}\right)^{\frac{1-\alpha^{2}}{1+\alpha^{2}}}-\frac{1}{3} \lambda r^{2}\left(1-\frac{r_{-}}{r}\right)^{\frac{2 \alpha^{2}}{1+\alpha^{2}}}\right] d t^{2} \\
& +\left[\left(1-\frac{r_{+}}{r}\right)\left(1-\frac{r_{-}}{r}\right)^{\frac{1-\alpha^{2}}{1+\alpha^{2}}}-\frac{1}{3} \lambda r^{2}\left(1-\frac{r_{-}}{r}\right)^{\frac{2 \alpha^{2}}{1+\alpha^{2}}}\right]^{-1} d r^{2} \\
& +r^{2}\left(1-\frac{r_{-}}{r}\right)^{\frac{2 \alpha^{2}}{1+\alpha^{2}}} d \Omega_{2}^{2},
\end{aligned}
$$

where $r_{+}, r_{-}$are two event horizons of the black hole. $\alpha$ is an arbitrary constant governing the strength of the coupling between the dilaton and the Maxwell field. The action with respect to the metric is

$$
\begin{aligned}
S= & \int d^{4} x \sqrt{-g}\left\{R-2 \partial_{\mu} \phi \partial^{\mu} \phi-e^{-2 \alpha \phi} F^{2}\right. \\
& \left.-\frac{2}{3} \lambda \frac{1}{\left(1+\alpha^{2}\right)^{2}}\left[\alpha^{2}\left(3 \alpha^{2}-1\right) e^{-2 \phi / \alpha}+\left(3-\alpha^{2}\right) e^{2 \phi \alpha}+8 \alpha^{2} e^{\phi \alpha-\phi / \alpha}\right]\right\} .
\end{aligned}
$$

When $\alpha=1$, the action restores to the Garfinkle-Horowitz-Strominger case. The corresponding dilaton field is

$$
e^{2 \alpha \phi}=e^{2 \alpha \phi_{0}}\left(1-\frac{r_{-}}{r}\right)^{\frac{2 \alpha^{2}}{1+\alpha^{2}}}, \quad e^{2 \alpha \phi_{0}}=\frac{r_{+} r_{-}}{\left(1+\alpha^{2}\right) Q^{2}} .
$$

It is apparent that three Liuoville-type potentials constitute the potential of the dilaton with respect to the cosmological constant.

\section{MULTI-DILATON-BLACK-HOLE SOLUTION IN DE SITTER UNIVERSE}

In what follows we shall, for completeness, present the multi-black hole solution in de Sitter universe. For extreme dilaton black hole, set $r_{+}=r_{-}=m / 4$, Eq.(26) becomes

$$
d s^{2}=-\left(1+\frac{m}{a r}\right)^{\frac{-2}{1+\alpha^{2}}} d t^{2}+a^{2}\left(1+\frac{m}{a r}\right)^{\frac{2}{1+\alpha^{2}}}\left(d r^{2}+r^{2} d \Omega_{2}^{2}\right)
$$

It closely resembles the Kastor-Traschen [21] solution and Gibbons-Kallosh [22] solution. Thus following the method developed by Kastor and Traschen, we obtain the cosmological multi-dilaton-black hole solution 


$$
\begin{aligned}
d s^{2} & =-\Omega^{\frac{-2}{1+\alpha^{2}}} d t^{2}+a(t)^{2} \Omega^{\frac{2}{1+\alpha^{2}}}\left(d x^{2}+d y^{2}+d z^{2}\right), \\
\Omega & =1+\sum_{i} \frac{m_{i}}{a r_{i}}, \quad r_{i}=\sqrt{\left(x-x_{i}\right)^{2}+\left(y-y_{i}\right)^{2}+\left(z-z_{i}\right)^{2}}, \quad a(t)=e^{H t},
\end{aligned}
$$

where $x, y, z$ are Cartesian coordinates on $R^{3}, m_{i}$ is the mass or charge of the $i$ th extremal black hole, and the charges must all be of the same sign. It is easy to find that when $H=0$, Eq.(31) restores to the Gibbons-Kallosh solution and when $\alpha=0$, Eq.(31) restores to the Kastor-Traschen solution.

\section{HORIZONS OF DILATON-DE SITTER SPACETIME}

Now let us make a discussion on the horizons of the dilaton-de Sitter spacetime. For simplicity in mathematics, we will concentrate on the case of the coupling constant $\alpha=1$. To this end, make variable transformation $r=D+\sqrt{x^{2}+D^{2}}$ in Eq.(13) and then rewrite the variable $x$ with $r$, we have

$$
\begin{aligned}
d s^{2}= & -\left(1-\frac{2 M}{D+\sqrt{r^{2}+D^{2}}}-H^{2} r^{2}\right) d t^{2} \\
& +\left(1+\frac{D^{2}}{r^{2}}\right)^{-1}\left(1-\frac{2 M}{D+\sqrt{r^{2}+D^{2}}}-H^{2} r^{2}\right)^{-1} d r^{2}+r^{2} d \Omega_{2}^{2}
\end{aligned}
$$

It is apparent there is no singularity in the metric and when the dilaton charge $D=0$, Eq.(32) is just the Schwarzschild-de Sitter solution. Horizons occur whenever $g_{00}=0$. This implies that

$$
1-\frac{2 M}{D+\sqrt{r^{2}+D^{2}}}-H^{2} r^{2}=0
$$

It is found that Eq.(33) gives only the cosmological horizon and the black hole event horizon. There is no inner Cauchy horizon present. This is different from the Reissner-Nordström-de Sitter case where three horizons are present.

The extremal dilaton black hole case, i.e. $M=D$, is of particular interest. Then Eq.(33) reveals that the black hole event horizon and the singularity disappear and only one cosmic horizon survives 


$$
r_{\cos }=\frac{1}{H} \sqrt{1-2 M H}
$$

This is also different from the extremal Reissner-Nordström-de Sitter case where two horizons are left. Provided that

$$
M=\frac{1}{27 H}\left[9 D H+8 D^{3} H^{3}+\left(3+4 D^{2} H^{2}\right) \sqrt{3+4 D^{2} H^{2}}\right], \quad 0<D H<\frac{1}{2},
$$

the black hole event horizon and the cosmological horizon coalesce. That is

$$
r_{b h, \cos }=\frac{1}{3 H} \sqrt{3-4 D^{2} H^{2}-2 D H \sqrt{3+4 D^{2} H^{2}}}
$$

Given that $D H=\frac{1}{2}$ in Eq.(36), then the only remaining horizon $r_{b h, c o s}$ also disappears. This reveals that the presence of dilaton has important consequences on the horizons of the black hole.

\section{CONCLUSION AND DISCUSSION}

To conclude, we have obtained the dilaton black hole solutions in the background of (anti) de Sitter spacetime with a new method we developed previously. The solution shows that the cosmological constant couples the dilaton field in a nontrivial way. The dilaton potential with respect to the cosmological constant includes three Liouville-type potentials. This is consistent with the discussion of Poletti and Wiltshire that no (anti) de Sitter version of dilaton black holes exist with only one Liouville-type potential. The resulting potentials are also consistent with the condition $\left.\frac{d V}{d \phi}\right|_{\phi=\phi_{0}}=0$ which is proposed by Poletti and Wiltshire for the existence of such solutions [18]. This type of derived potential can be obtained when a higher dimensional theory is compactified to four dimensions, including various super gravity models (see[24] for a recent discussion of these aspects) [23]. In particular, when the coupling constant $\alpha= \pm \sqrt{\frac{1}{3}}, \pm 1, \pm \sqrt{3}$, the potential is just the SUSY potential.

We also found the coordinate transformation which recasts our solution in the Schwarzschild coordinate system. Some discussions on the horizons in the end show that the dilaton has important consequences on the property of the black hole. The supersymmetry 
properties of the charged dilaton (anti) de Sitter black holes as well as their relevance to the Ads/CFT correspondence are currently under study.

\section{ACKNOWLEDGMENTS}

This study is supported in part by the Special Funds for Major State Basic Research

Projects and by the National Natural Science Foundation of China. SNZ also acknowledges supports by NASA's Marshall Space Flight Center and through NASA's Long Term Space Astrophysics Program. 


\section{REFERENCES}

[1] G. W. Gibbons, K. Maeda, Nucl. Phys. B 298 (1988) 741.

[2] D. Garfinkle, G. Horowitz, A. Strominger, Phys. Rev. D 43 (1991) 3140.

[3] D. Brill, G. Horowitz, Phys. Lett. B 262 (1991) 437.

[4] R. Gregory, J. Harvey, Phys. Rev. D 47 (1993) 2411.

[5] T. Koikawa, M. Yoshimura, Phys. Lett. B 189 (1987) 29.

[6] D. Boulware, S. Deser, Phys. Lett B 175 (1986) 409.

[7] M. Rakhmanov, Phys. Rev. D 50 (1994) 5155.

[8] B. Harms, Y. Leblanc, Phys. Rev. D 46 (1992) 2334.

[9] C. F. E. Holzhey, F. Wilczek, Nucl. Phys. B 380 (1992) 447.

[10] J. M. Maldacena, Adv. Theor. Math. Phys. 2 (1998) 231.

[11] J. M. Maldacena, Int. J. Theor. Phys. 38 (1999) 1113.

[12] E. Witten, Adv. Theor. Math. Phys. 2 (1998) 253.

[13] D. Klemm, W. A. Sabra, Phys. Lett. B 503 (2001) 147.

[14] S. S. Gubser, I. R. Klebanov, A. M. Polyakov, Phys. Lett. B 428 (1998) 105.

[15] O. Aharony, S. S. Gubser, J. M. Maldacena etc. Phys. Rep. 323 (2000) 183.

[16] R. Bousso, S. W. Hawking, Phys. Rev. D 57 (1998) 2436.

[17] C. M. Hull, JHEP 9807 (1998) 021.

[18] S. J. Poletti, D. L. Wiltshire, Phys. Rev. D 50 (1994) 7260.

S. Mignemi and D. L. Wiltshire, Phys. Rev. D46 (1992) 1475.

[19] T. Okai, hep-th/9406126; G. Clement and C. Leygnac, gr-qc/0405034; Rong-Gen Cai 
and An Zhong Wang 2004 Phys. Rev. D70 084042; Rong-Gen Cai and Yuan Zhong Zhang 2001 Phys. Rev. D64 104015; Rong-Gen Cai, Jeong-Young Ji and Kwang-Sup Soh 1998 Phys. Rev. D57 6547; Rong-Gen Cai and Yuan-Zhong Zhang 1996 Phys. Rev. D54 4891.

[20] C. J. Gao, S. N. Zhang, "Arbitrary Dimensional Reissner-Nordström-FRW metric" submitted to Phys.Lett.B.

[21] D. Kastor, J. Traschen J, Phys. Rev. D 47 (1993) 5401.

[22] G. W. Gibbons, R. E. Kallosh, Phys. Rev. D 51 (1995) 2839.

[23] E. Radu, D. H. Tchrakian, hep-th/0410154.

[24] S. B. Giddings, Phys. Rev. D 68 (2003) 026006. 